\title{
The Manchester procedure combined with laparoscopic sacrohysteropexy by retroperitoneal tunneling
}

\author{
(D) Kerem Doğa Seçkin¹, (D) Pınar Kadiroğulları², (D) Hüseyin Kıyak1 , (D) Ali Rıza Doğan³, (D Ömer Lütfi Tapısız ${ }^{3}$ \\ ${ }^{1}$ Clinic of Obstetrics and Gynecology, University of Health Sciences Turkey, Kanuni Sultan \\ Süleyman Training and Research Hospital, İstanbul, Turkey \\ ${ }^{2}$ Clinic of Obstetrics and Gynecology, Acıbadem Mehmet Ali Aydınlar University, Atakent \\ Hospital, İstanbul, Turkey \\ ${ }^{3}$ Clinic of Obstetrics and Gynecology, University of Health Sciences Turkey, Ankara Etlik Zübeyde Hanım Women's Health \\ Training and Research Hospital, Ankara, Turkey
}

\section{Abstract}

This video will demonstrate a minimally invasive technique, in which the Manchester procedure was combined with laparoscopic sacrohysteropexy by retroperitoneal tunneling in patients with uterine prolapse and cervical elongation who wished to preserve the uterus. The principle steps and techniques to complete the operation are dictated in the video. The prolapse surgery was performed uneventfully, and the uterus was restored to its anatomical position. During the two years of follow-up, there were no complications from the prolapse or mesh-related events. No prolapse recurrence was observed. This technique facilitates uterine-sparing surgery, results in less bleeding and shorter operative time, and we believe that it may reduce the recurrence of prolapse due to the elongation of the cervix. (J Turk Ger Gynecol Assoc 2022; 23: 219-21)

Keywords: Cervical elongation, Manchester procedure, sacrohysteropexy

Received: 12 February, 2021 Accepted: 17 May, 2021

\section{Introduction}

Approximately half of advanced-stage uterine prolapse (46.1\%) is accompanied by cervical elongation (1). There is an increased desire for uterine preservation in pelvic organ prolapse (POP) surgery, and cervical elongation can limit this possibility. Hysteropexy can result in less bleeding and take less time to complete, thus reducing anesthesia time. The risk of mesh erosion is five times lower in hysteropexy than in hysterectomy (2). However, the risk of reoperation is greater in hysteropexy (2). The technique described herein, which is a combination of two procedures, is recommended to the patient who desires to preserve the uterus, wants to minimize the risk of possible recurrence, and also has an active sexual life.

\section{Materials and surgical technique}

The 38-year-old patient was gravidity 3 , parity 3 and had a grade-2 elongation of the uterine cervix (4) and stage-3 apical (uterine) prolapse. The Pelvic Organ Prolapse Quantification System (POP-Q) findings were as follows: Aa $-1, \mathrm{Ba}-2, \mathrm{C}+2$, $\mathrm{D}+2$, Ap -2, Bp -3. The patient wished to preserve the uterus. (Supplementary Video S1).

Manchester procedure: The length of the cervix up to the sacrouterine ligament-uterus connection was measured as approximately $7-8 \mathrm{~cm}$ (Figure 1). A circular incision in the vagina was performed and ligated with bilateral cardinal ligaments (5). The extra-prolonged cervix was amputated (Figure 2), leaving $3-3.5 \mathrm{~cm}$ of the cervix intact. The vaginal mucosa was prepared in flap form, and the posterior vaginal mucosa was cut to the sacrouterine level. 
Laparoscopic sacrohysteropexy: A $10 \mathrm{~mm}$ trocar was inserted into the abdomen by direct entry technique from the umbilicus, and a pneumoperitoneum was created. Two lateral ports were placed on bilateral lower quadrants, and one suprapubic trocar was placed in the same plane as one lateral trocar.

- Peritoneal incision: The location of the promontory was determined anatomically by laparoscopy, and an incision was performed on its peritoneum. The incision was enlarged caudally and distally. The loose connective tissue on the promontory was dissected and the anterior longitudinal ligament was uncovered.

- Sigmorectal colon suspension: The sigmorectal colon was suspended with a T-Lift device (Vectec $^{\mathrm{TM}}$, France) for better visualization.

- Retroperitoneal tunneling: The remaining cervical tissue was held with a tenaculum. Tunneling was performed with ring forceps under the cervical tissue above the vaginal tissue retroperitoneally, from the point where the right sacrouterine ligament adheres the uterus to the right pelvic sidewall, under camera guidance. In order to avoid unintentional damage to adjacent anatomical structures, ring forceps with atraumatic and blunt ends were used. The vaginal mucosa was opened to the peritoneal incision, which was created earlier by laparoscopy extending to the cervix. The previously created peritoneal area reached the cervix and the vaginal mucosa was opened up to the incision previously prepared by laparoscopy.

- Preparation of the mesh: a 4x15 cm synthetic macroporous polypropylene mesh segment (Düzey Medikal, İstanbul, Turkey) was prepared and inserted through the umbilical trocar into the abdomen, and one side of the mesh was grasped by a vaginally-inserted forceps (Figure 3 ).

- Suturing of the mesh to the posterior cervix: Propilen ${ }^{\circledR}$ polypropylene synthetic, non-absorbable, monofilament, no: 1 suture (Doğsan, İstanbul, Turkey) was used.

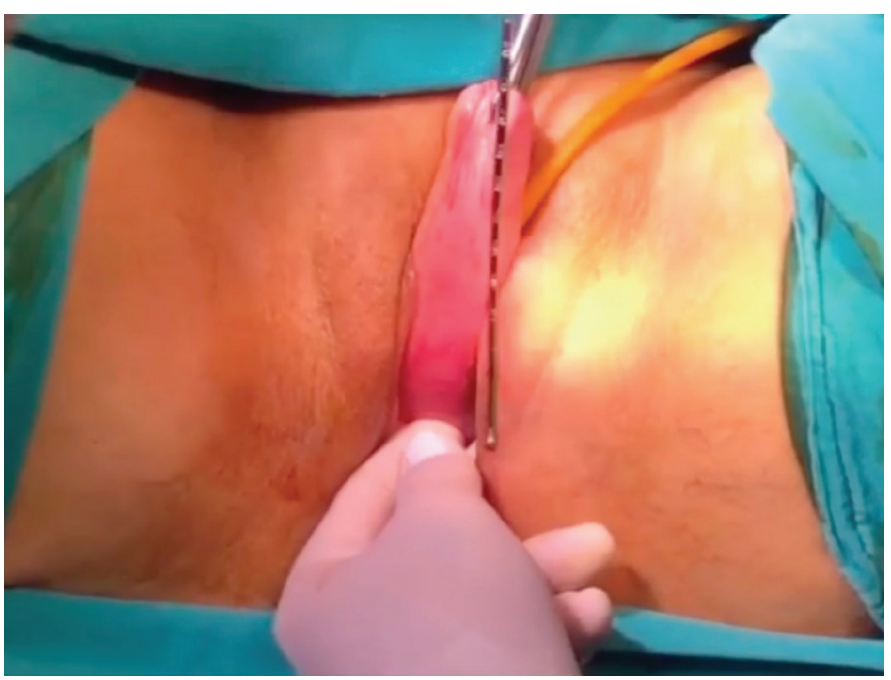

Figure 1. The measurement of the length of the cervix
- The Manchester procedure was completed with reattachment of the cardinal ligament, using Sturmdorf sutures.

- Tailoring the position of the uterus: The cervix was positioned at the $-5,-6$ level. Before fixing the mesh to the anterior longitudinal ligament, the cervix was confirmed to be at the $-5,-6$ level by hysterometry. After that measurement, the mesh was fixed to the ligament. We used five non-absorbable tackers to fix the mesh.

- Anchoring of the upper pole of the mesh to the anterior longitudinal ligament was accomplished with a $5 \mathrm{~mm}$ laparoscopic ProTack ${ }^{\mathrm{TM}}$ Fixation Device (Medtronic, Minneapolis, MN, USA).

The peritoneum was closed with a continuous running 2-0 absorbable suture line. The whole mesh was peritonized to avoid adhesion to or extrusion from the bowel. Reperitonization was performed with attention to the ureter. The T-Lift device was removed, and the ureter was visualized and inspected to ensure no damage had occurred, completing the process.

The procedure was completed successfully with a total operative time of 78 minutes and estimated blood loss of 180 $\mathrm{mL}$. The uterine prolapse was corrected (postoperative POP-Q findings: Aa -1, Ba -3, C -5, TVL 7.5, Ap -2, Bp -3, D -7.5) (Figure 4). No intraoperative surgical complications were observed. On postoperative day 2 , recovery had been uneventful and the patient was discharged from the hospital. During two years of follow-up, no recurrence, complications of mesh exposure, de novo stress urinary incontinence or bowel obstruction have occurred. The patient had reported that she had no plan for pregnancy before surgery.

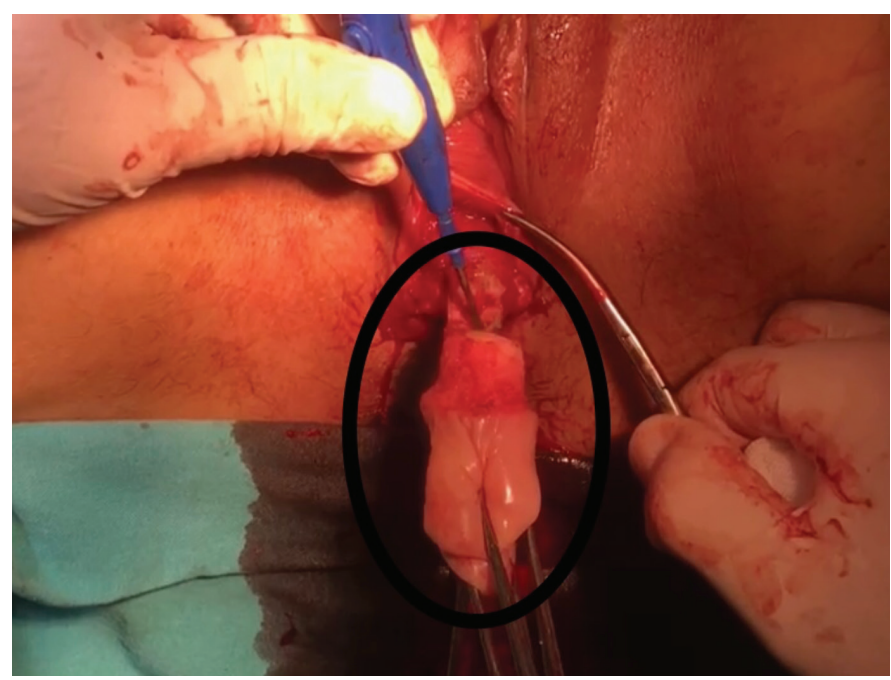

Figure 2. The view of elongated cervix at the time of incision 


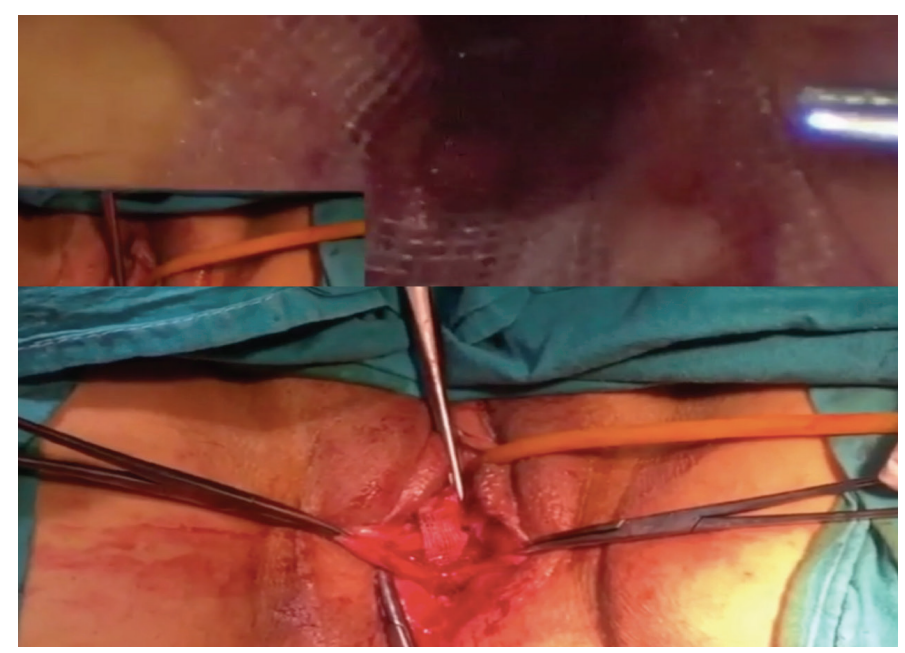

Figure 3. The appearance of the mesh by vaginal and abdominal route

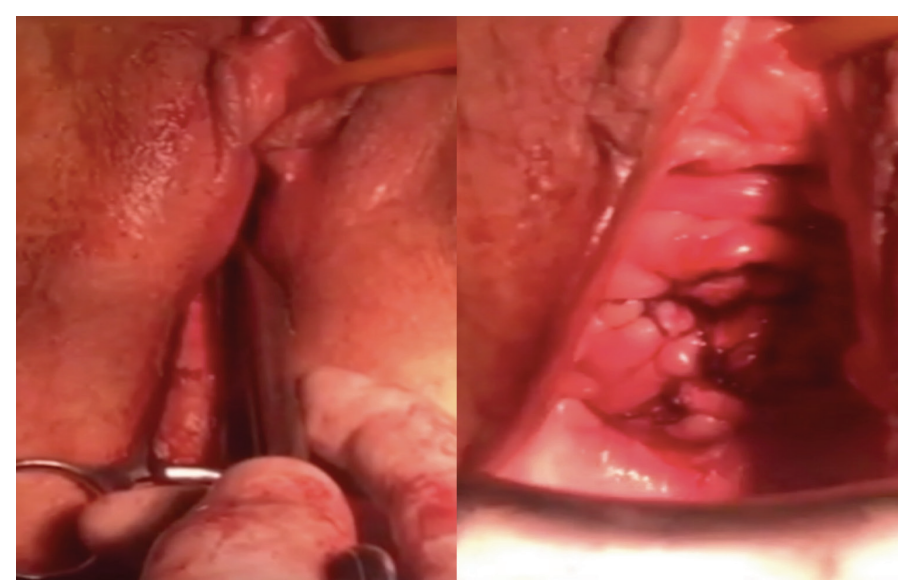

Figure 4. The depth of the cervix and the last view after operation
Supplementary Video S1. The Manchester procedure was combined with laparoscopic sacrohysteropexy

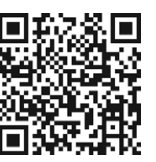

https://www.doi.org/10.4274/jtgga.galenos.2021.2021.0029.video1

Informed Consent: Written informed consent was obtained from the patient for publication of this video article and any accompanying images

Conflict of Interest: No conflict of interest is declared by the authors.

Financial Disclosure: The authors declared that this study received no financial support.

\section{References}

1. Jefferis H, Price N, Jackson S. Laparoscopic hysteropexy: 10 years' experience. Int Urogynecol J 2017; 28: 1241-8.

2. Hsiao SM, Chang TC, Chen CH, Li YI, Shun CT, Lin HH. Risk factors for coexistence of cervical elongation in uterine prolapse. Eur J Obstet Gynecol Reprod Biol 2018; 229: 94-7.

3. de Oliveira SA, Fonseca MCM, Bortolini MAT, Girão MJBC, Roque MT, Castro RA. Hysteropreservation versus hysterectomy in the surgical treatment of uterine prolapse: systematic review and meta-analysis. Int Urogynecol J 2017; 28: 1617-30.

4. Mothes AR, Mothes H, Fröber R, Radosa MP, Runnebaum IB. (Systematic classification of uterine cervical elongation in patients with pelvic organ prolapse. Eur J Obstet Gynecol Reprod Biol 2016; 200: 40-4.

5. Thys SD, Coolen A, Martens IR, Oosterbaan HP, Roovers J, Mol B, et al. A comparison of long-term outcome between Manchester Fothergill and vaginal hysterectomy as treatment for uterine descent. Int Urogynecol J 2011; 22: 1171-8. 